\title{
A Simulation Model for Industrial Multi-Channel Wireless Sensor Networks
}

\author{
Ruan D. Gomes*, Diego V. Queiroz*, Iguatemi E. Fonseca, and Marcelo S. Alencar
}

\begin{abstract}
The use of Wireless Sensor Networks (WSN) in industrial environments is subject to problems, such as shadowing and fading. In addition, the wireless channel in many industrial environments is non-stationary for a long term, which can cause abrupt changes in the characteristics of the channel over time. A way to deal with those problems is the use of multi-channel protocols. However, it is difficult to evaluate, and compare different approaches because there is no reliable simulation model for industrial environments. This paper presents experimental results that characterize the wireless channel in industrial environments, and proposes a simulation model that captures the effects of fading, shadowing, and the non-stationary characteristics of the channel. It also considers the differences in the behavior of the different channels, and the asymmetry of the links. The model was integrated into the open source simulator Castalia. After the integration, two simulation studies were performed with the proposed model. In the first one, the Tree-based Multi-Channel Protocol was implemented and evaluated, and a comparison was made with the default model from Castalia. In the second case, CSMA/CA protocol, as defined in IEEE 802.15.4 standard, was compared to a protocol based on Time-Slotted Channel Hopping (TSCH) mode of IEEE 802.15.4e standard. The results showed that the use of TDMA and channel hopping is an alternative to deal with the problems of wireless channels in industrial environments.
\end{abstract}

Index Terms-Industrial Wireless Sensor Networks, Multi-Channel Protocols, Simulation.

\section{INTRODUCTION}

$\mathbf{T}$ HE use of Wireless Sensor Networks (WSN) to implement systems for monitoring and control in industrial environments presents some advantages, when compared to the use of wired networks, such as low cost and high flexibility. However, it is necessary to deal with interference problems, possibly caused by other wireless networks, and with the large and small-scale fading, due to the presence of many objects and obstructions in the environment (usually made with metallic materials) [1].

The Associate Editor coordinating the review of this manuscript and approving it for publication was Prof. José Cândido Silveira Santos Filho.

Ruan D. Gomes is with the Federal Institute of Education, Science, and Technology of Paraíba, Guarabira, Brazil, and with the Post-Graduate Program in Electrical Engineering - PPgEE - COPELE, Campina Grande, Brazil, e-mail: (ruan.gomes@ifpb.edu.br).

Diego V. Queiroz and Iguatemi E. Fonseca are with the Informatics Center, Federal University of Paraíba, João Pessoa, Brazil, e-mail: (diego@sti.ufpb.br, iguatemi@ci.ufpb.br).

Marcelo S. Alencar is with the Department of Electrical Engineering, Federal University of Campina Grande, Campina Grande, Brazil, e-mail: (malencar@dee.ufcg.edu.br).

*Diego V. Queiroz and Ruan D. Gomes contributed equally to this paper. They are also in a doctorate mobility at Universidad Politécnica de Madrid, Spain.

Digital Object Identifier: 10.14209/jcis.2017.4.
In industrial environments, differences on the characteristics of the channels may be observed, related to the multipath profile of the environment, since the channels are uncorrelated in frequency, and the impact of multipath is different for different channels [2]. In addition, changes in the topology of the environment (e.g., movement of a large metal structure) and reflections may cause changes in channel characteristics over time, which may lead to differences in the mean value of the received power and in the variance, even for static nodes. Thus, when the wireless channel is analyzed in the long run, it is possible to observe abrupt changes in its profile at certain times [3].

The development of multi-channel protocols is an alternative to deal with the variations that occur in the quality of the channels over time. These protocols allow a better channel utilization, increasing the overall throughput of the network through simultaneous transmissions. It is also possible to deal with the spatial variations in channel quality for different segments of the network. Some solutions use multiple channels simultaneously, with multiple transceivers in the sensor nodes, or using different channels in different segments of the network [4]. Other approaches use frequency hopping, such as Medium Access Control (MAC) protocols of WirelessHART, ISA100.11a, and IEEE 802.15.4e standards [5] [6]. Some works focus on dynamic channel allocation mechanisms [7] [8], in which the WSN changes the channel when its quality decreases.

To simulate or emulate the characteristics of multiple channels simultaneously, in order to compare different multi-channel protocols, it is recommended the use of a common testbed and simulators that are more accurate. Some papers describe simulation studies of Industrial WSN (IWSN) [9] [10] [11] [12], but they consider neither the non-stationary characteristics of the wireless channel for a long term, nor the uncorrelation between different channels.

This paper describes a simple and reliable model to simulate multi-channel protocols for IWSN that captures the effects of fading, shadowing, and the non-stationary characteristics of the channel. It also considers the differences in the characteristics of the different channels, and the asymmetry of the links. This model was first described in the conference paper [13], and was also used to evaluate link quality estimators for an IWSN in [14]. Some experimental results, that demonstrate the characteristics of the wireless channel, are also presented in this paper. The experiments were conducted using IEEE 802.15.4 radios in industrial environments.

The model described in [13] is extended to support the simulation of multi-channel protocols, and to capture the 
asymmetry of the links. It was integrated into the open source simulator Castalia [15], and two simulation studies were performed to evaluate the performance of multi-channel protocols in industrial environments. In the first one, the Tree-based Multi-Channel Protocol (TMCP) was implemented and evaluated. In TMCP, the network is statically divided into disjoint subtrees to reduce collisions, and different channels are allocated to each sub-tree. A comparison study was made using both the proposed model and the default one of the simulator. In the second one, the single-channel Carrier Sense Multiple Access with Collision Avoidance (CSMA/CA) protocol, as defined in IEEE 802.15.4 standard, is compared to a protocol based on Time-Slotted Channel Hopping (TSCH) mode of IEEE 802.15.4e standard, which uses Time Division Multiple Access (TDMA) and frequency hopping.

The results showed that static protocols are not adequate to implement IWSN, since they are not capable to deal with the variations that occur in the quality of the channels over time. The use of TDMA and channel hopping is an alternative to deal with the problems that affect the quality of the channels in industrial environments, and to reduce the number of collisions. However, some challenges need to be addressed, such as the time synchronization. Different approaches can be evaluated using the simulation model proposed in this paper, to support the development of novel protocols appropriate for IWSN.

\section{THE WIRELESS CHANNEL IN INDUSTRIAL ENVIRONMENTS}

The industrial environments usually contain metallic and mobile objects, such as robots, cars and people, which influence both the large-scale path loss and the multipath fading. In [1], experiments were conducted to analyze the path loss and shadowing in an industrial environment in frequencies of $900 \mathrm{MHz}, 2400 \mathrm{MHz}$, and $5200 \mathrm{MHz}$. Equation 1 shows the path loss in $\mathrm{dB}$ at distance $d$ between transmitter and receiver [16],

$$
L(d)=L\left(d_{0}\right)+10 n \log \left(\frac{d}{d_{0}}\right)+X_{\sigma},
$$

in which $n$ is the path loss exponent, and $d_{0}$ is the reference distance. There is a variation in the received power, depending on where the measurement is performed. To capture this variation, a random variable $X \sigma$ is included in Equation 1, in which $X \sigma$ is a Gaussian distribution with zero mean and standard deviation $\sigma$, and the two parameters are represented in $\mathrm{dB} . X \sigma$ is known as log-normal shadowing. In [1], the values of the aforementioned parameters were obtained for different scenarios in an industrial environment. Castalia simulator implements log-normal shadowing model.

Besides large-scale path loss and shadowing, the small-scale attenuation also needs to be considered, due to rapid changes in the multipath profile of the environment caused by the movement of objects around the receiver and transmitter. Experiments have shown that in industrial environments the temporal attenuation follows the Rice distribution [1]. This distribution models the small-scale fading when there is a dominant stationary signal and random components overlapping with the main component. In industrial environments, there are usually several invariant rays, and just a small portion of multipath profile is affected by moving objects [1]. The Castalia simulator does not implement small-scale fading with the Rice distribution, or any other probability distributions.

The probability density function that describes the envelope of a signal under Ricean fading is

$$
p_{R}(x)=\frac{x}{b^{2}} e^{-\frac{\left(x^{2}+A^{2}\right)}{2 b^{2}}} \mathrm{I}_{0}\left(\frac{A x}{b^{2}}\right), x \geq 0,
$$

in which $\mathrm{I}_{0}(\bullet)$ is the modified Bessel function of the first kind with order zero, $A^{2}$ is the power of the dominant stationary signal, and $2 b^{2}$ is the average power of the random multipath components.

\section{A. Fading in Industrial Environments in Long Term}

The wireless channel can be modeled as wide-sense stationary for a short term. However, the channel properties can change significantly in a period of a few hours, due to modifications in the topology of the environment. These changes are not taken into account by usual probability distributions that are used to model fading channels.

The characterization of the wireless channel in an industry was performed for a long period (20 hours) in [3]. The results showed that Rice distribution only fits the received power for small periods, in which the mean value of the received power remains constant. For example, the movement of a big metallic structure can change the multipath profile related to a set of rays that remained invariant for a long term, which causes differences on the mean value of the received power, although the transmitter and receiver remain static. Experiments described in [3] showed that abrupt changes could occur in the link characteristics. In that experiment, the received power varied around $-55 \mathrm{dBm}$ during seven hours, and after this period, the mean value of the received power changed abruptly to $-46 \mathrm{dBm}$. An experiment described in [17] also presented similar behavior.

In experiments performed by the authors of this paper, a similar behavior was also observed, as can be seen in Figure 1, that shows the Received Signal Strength Indicator (RSSI)

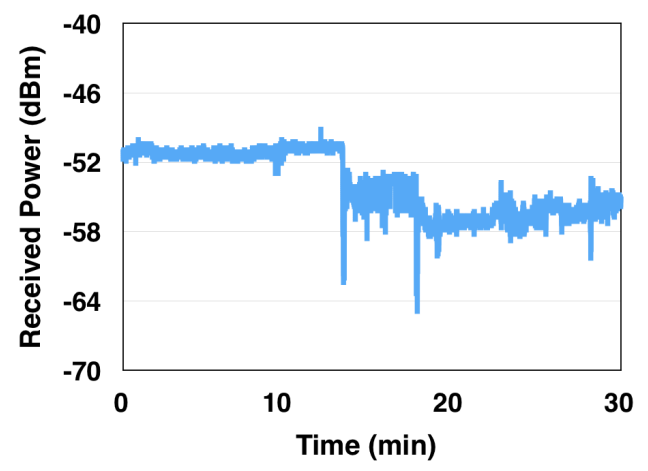

Fig. 1. Non-stationary behavior of the channel in an industrial environment. 


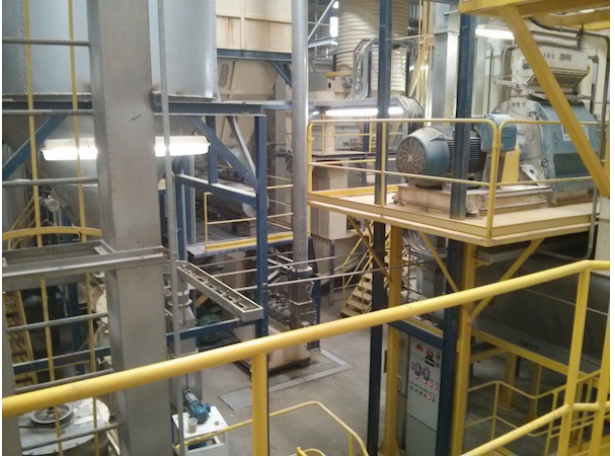

Fig. 2. Industry where the experiments were performed.

values acquired, during 30 minutes, from an experiment conducted with two IEEE 802.15.4 radios operating in $2.4 \mathrm{GHz}$ band, in an industrial environment (Figure 2). RSSI is a metric provided by the transceiver to assess the received power. An abrupt change in the channel characteristics occurs at a certain time, which indicates that the channel is non-stationary in the long run.

Agrawal et al. [18] used a composite distribution to capture both shadowing, and fading for a long term. The model is called Nakagami- $m / \log$-normal. The parameter of Nakagami- $m$ distribution defines the level of fading, and the parameters of log-normal distribution define the effects of shadowing. The validation of the distribution was performed using a set of values obtained from experiments in industrial environments, and considering the Cumulative Distribution Function (CDF) of Rice, Rayleigh, Nakagami- $m$, log-normal, and Nakagami- $m /$ Log-normal. The validation was based on the inferior tail, since this region is responsible for most transmissions errors. It was shown that the usual distributions that model fading overestimate the occurrence of deep fading. Log-normal distribution presented a better fit with the experimental values, but this distribution underestimates the occurrence of deep fading. Nakagami- $m / \log$-normal presented a better result, since it can capture the effects of shadowing and fading for a long term.

Wang et al. [19] applied the Bayesian Change Point Detection (CPD) on Nakagami- $m$ channel model to capture the abrupt changes in time that occurs in the quality of the channel for a long term. The CPD identifies when the probability distribution of a stochastic process changes. To validate the model, sequences of values using Nakagami- $m$ model were generated, and a manual division of segments was performed, in which each segment presents different values for the distribution parameter. Experiments were performed and the segments were detected in the trace generated by the experiment. Then, the parameters for Nakagami- $m$ distribution in each segment were calculated.

Although the authors in [18] [19] have described models to capture the modifications in the channel characteristics for a long term, the models are adequate only for analytical evaluation of WSN, since the instantaneous random values generated following the proposed distributions do not correspond to the observed in practice, although the CDF of the model presents a good fit with the CDF of the experimental data.

\section{B. Coherence Bandwidth}

IEEE 802.15.4, which is the usual communication standard for WSN, defines sixteen channels in $2.4 \mathrm{GHz}$ band, with $2 \mathrm{MHz}$ of bandwidth, and $5 \mathrm{MHz}$ of channel spacing. However, some standards, such as WirelessHART, uses only 15 channels [20]. Experiments described in [21] showed that changing the communication channel can lead up to a $30 \mathrm{~dB}$ of difference in the received power inside an office environment. Varga et al. [22] performed experiments for a short range in an environment without multipath, and with Line-Of-Sight (LOS). In that experiment, differences up to $10 \mathrm{~dB}$ were observed for some channels. In the experiments described in [23], performed in an office environment, even the adjacent channels were uncorrelated, for distances greater than 6.5 meters. This difference may be larger in channels with high Root-Mean-Square (RMS) delay spread, as can occur in many industrial environments.

Another set of experiments was performed by the authors of the present paper to verify the correlation between the channels. Figure 3 shows the received power calculated using RSSI of the received packets for nine different channels. The values were filtered using an average filter, with a window of 20 samples, to better analyze the shadowing in each channel. The node received packets in each channel during three minutes, with rate of one packet per second. The transmitter was positioned 23 meters (Figure 4) away from the receiver without LOS. The nodes used in this experiment have a MRF24J40MA transceiver, a Printed Circuit Board (PCB) antenna with $2.09 \mathrm{dBi}$ of gain, and $0 \mathrm{dBm}$ of transmit power. The nodes were configured remotely using another node, designed to communicate to each node the information of the channel to be used in the next replication. This was done to avoid a change in position of the nodes involved in the experiment.

The result shows that the multipath profile of the environment affects differently each channel. For example, Channels 11 and 13 have shown a difference of about $15 \mathrm{~dB}$ in the mean value of received power.

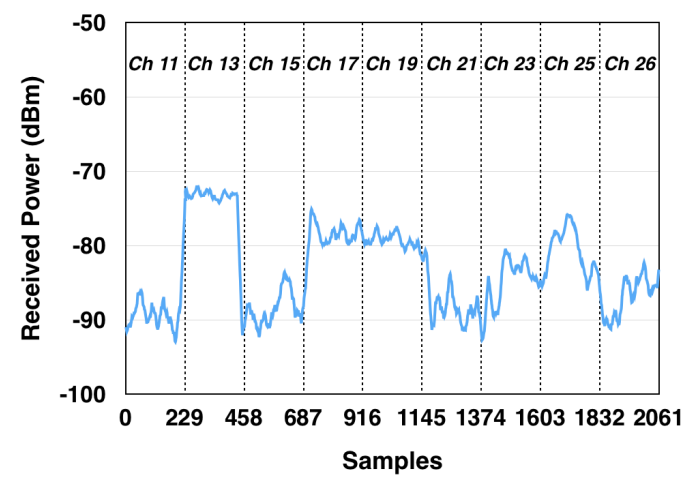

Fig. 3. Experiment results with uncorrelated channels.

Another experiment was performed to investigate the Packet Reception Rate (PRR) for different channels, considering 


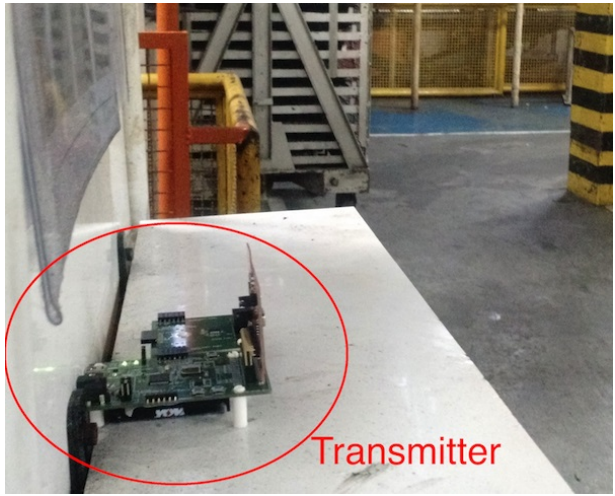

Fig. 4. Environment where the experiment was performed.

static nodes, in the industry shown in Figure 4. Two nodes (transmitter and receiver) were configured to operate using channel 11, and the transmitter was configured to transmit packets during 60 seconds. After that, the nodes switched to the channel 19 and the transmitter sent packets to the receiver during 60 seconds. The transmitter and the receiver were positioned $30 \mathrm{~m}$ apart without LOS. Figure 5 shows the result of this experiment. The PRR was $91 \%$ when using channel 11 , and $51 \%$ when using channel 19 . It is possible to notice that, even in the same environment, distance, and nearly at the same time, the channels also showed different characteristics for this experiment.

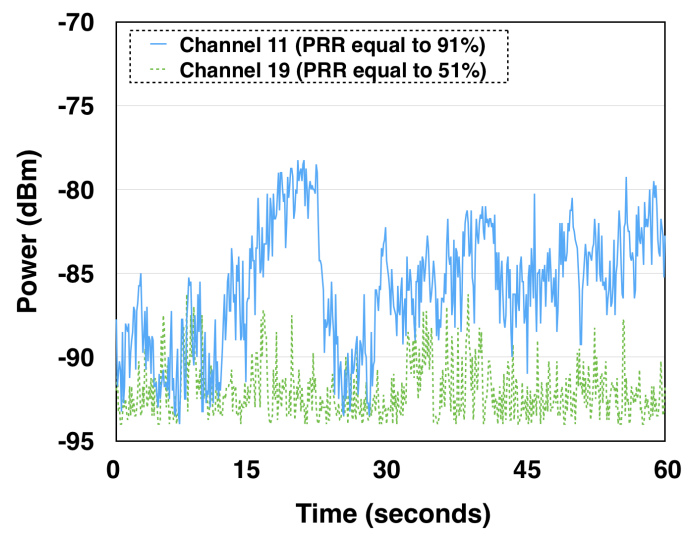

Fig. 5. RSSI values obtained in experiments using different channels in an industrial environment.

\section{Multi-channel Protocols}

An alternative to deal with the issues discussed in Section II is the development of multi-channel protocols. The use of such protocols allows an increase on the network transmission capacity, through simultaneous transmissions in several different channels. It is also possible to deal with the spatial variations in the quality of the channels [24].

The static Tree-based Multi-Channel Protocol (TMCP) [25] was chosen to evaluate the model described in this paper. It has three components: Channel Detection (CD), Channel Assignment (CA), and Data Communication (DC). The CD module finds orthogonal channels that can be used. Two nodes are used to sample the quality of each channel. Among the channels with good quality, the non-adjacent channels are prioritized.

The CA module partitions the network into $k$ subtrees and defines a channel for each one, exploiting the parallel transmissions among them. The intra-tree interference cannot be avoided completely, but when the network is partitioned, the interference between the nodes decreases, improving the overall Quality of Service (QoS) of the network. After assigning the channels, the DC module manages the data collection in each sub-tree. When a node has a packet to transmit to the sink-node, it uses the routers in its subtree and the channel allocated for the subtree. This protocol considers that the sink-node has a network interface for each channel. This approach works well with a small number of channels and has a simple transmission scheme, without the need for synchronization of the nodes, which makes it suitable for WSN. In order to assess the real benefits of these multi-channel protocols, and to develop novel protocols for IWSN, it is necessary to use network simulators with accurate models, such as the simulation model described in this paper.

Some standards have been proposed in the last years with focus on industrial applications, such as WirelessHART and ISA100.11a. Both WirelessHART and ISA100.11a are based on the physical layer of IEEE 802.15.4, but defines its own MAC layer. Instead of using CSMA/CA, as defined by IEEE 802.15.4 standard, they use a MAC layer with TDMA. By using TDMA, collisions are avoided and the power consumption can be optimized. They also use frequency hopping and blacklisting, to mitigate the problems related to interference and fading. However, without an adequate management of the blacklist, the communication performance may be low for these standards [26].

Recently, the IEEE $802.15 .4 \mathrm{e}$ standard was released, with the goal of proposing solutions for applications that require high reliability, such as industrial applications [6]. Five modes of operation are defined, that is: Time-Slotted Channel Hopping (TSCH), Deterministic and Synchronous Multi-Channel Extension (DSME), Low Latency Deterministic Network (LLDN), Asynchronous Multi-Channel Adaptation (AMCA), and Radio Frequency Identification Blink (BLINK). However, only TSCH, DSME, and LLDN modes have been explored in the literature until recently. In general, the modes of IEEE 802.15.4e are based on TDMA or frequency hopping to reduce collisions and mitigate the effects of interference and fading, and to satisfy the requirements of industrial applications in terms of reliability and determinism.

One of the main differences between the new standard IEEE $802.15 .4 \mathrm{e}$ in comparison to the previous IEEE 802.15.4 standard is the use of multiple channels. Besides using TDMA, TSCH mode employs channel hopping. When using this mechanism, the nodes usually switch to a new channel before each transmission, which makes the network more robust against problems that affect only a subset of the channels.

\section{Non-Stationary Simulation Model FOR IWSN}

Castalia is a discrete-event simulator developed in $\mathrm{C}++$, based on the open-source simulation framework OMNeT++. 
Its radio module is based on real radios for embedded low-power devices, including support to the transceivers CC2420 and CC1000, and log-normal shadowing model, which provides precise estimates for the average path loss. Castalia is also more specific for low-rate networks than other simulators, such as Network Simulator 3 (NS3) and OPNET, which focus on general networks.

To allow the simulation of multi-channel protocols for IWSN, it is necessary to use a model that allows capturing the characteristics of the channels for a long term and the simulation of multiple channels simultaneously. Thus, the non-stationary behavior of wireless channel, and the uncorrelation of different channels need to be considered, as explained in Section II.

The fading effect on the channel, in the long run, that is proposed in this paper, can be modeled as a two-state Markov chain, illustrated in Figure 6.

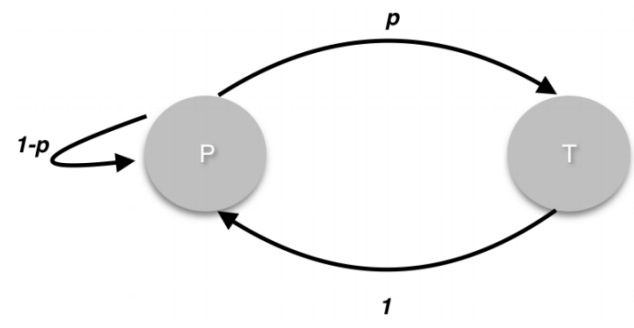

Fig. 6. Markov chain with two states.

While the Markov chain remains in the $P$ state, the channel characteristics remain unchanged. The received power values for a certain link between two nodes are generated considering a signal under Ricean fading. The large-scale path loss is modeled using log-normal shadowing model.

The transition to the state $T$ occurs with probability $p$. When this occurs, the parameters of the channel model are modified, and therefore an abrupt change occurs in the quality of the channels. After changing the parameters of the models, the Markov chain returns to the state $P$ with probability one.

A mean time of change $\left(T_{c}\right)$, in minutes, is defined for the model, which is used to define the value of $p$. In the implementation of the model, the rate of change of the Markov chain was set to one minute, thus $p=\frac{1}{T_{c}}$. With this parameter, it is possible to simulate environments that remain unchanged for a long term, and environments that present frequent changes in the topology. The simulation results obtained using the model is compatible with results from experiments performed in industrial environments [2] [17] [18]. In this paper, Rice distribution was used to model fading, but other distributions could be used, such as Nakagami- $m$ distribution. The integration of other distributions will be implemented in the future.

The first step to redefine the parameters of the channel, when the Markov chain reaches the state $T$, is the definition of the mean power of reception $\left(P_{R}(d, t)_{\mathrm{dBm}}\right)$ for a distance $d$ and time $t$, according to

$$
P_{R}(d, t)_{\mathrm{dBm}}=P_{T}-L(d),
$$

in which $P_{T}$ is the power transmission and $L(d)$ is calculated using Equation 1. Considering the same distance between transmitter and receiver, $P_{R}(d, t)_{\mathrm{dBm}}$ presents a random variation regarding $X_{\sigma}$, which can increase or decrease the average value of the received power.

The instantaneous value of reception power is obtained by adding the value of $P_{R}(d, t)_{\mathrm{dBm}}$ to the fading value, which is obtained by simulation of a channel under Ricean fading. Experiments described in [1] validated the use of Rice distribution as the model for temporal attenuation, for fixed location in industrial environments. In [1], experiments were conducted to determine the Ricean factor $(K)$ for different scenarios. These values were used as parameters for the simulations described in this paper.

The Ricean factor, in $\mathrm{dB}$, is defined as

$$
K=10 \log \left(\frac{A^{2}}{2 b^{2}}\right),
$$

in which $K$ is the ratio of the power in the LOS component $\left(A^{2}\right)$ to the power in the other (non-LOS) components $\left(2 b^{2}\right)$ [27].

To generate the random values following Rice distribution, a $\mathrm{C}++$ library called IT $++{ }^{1}$ was used, which is a library that can be used to simulate communication systems. This library implements the distribution to simulate the transmission of signals under Ricean fading.

To model the variations in the level of small-scale fading, it is also possible to configure a value for the standard deviation of $K$ factor $\left(K_{\sigma}\right)$. Thus, every time a change in the channel characteristics occurs, a new value of $K$ is defined, using a normal distribution with the mean value of $K$ and the standard deviation $K_{\sigma}$ as parameters. In experiments performed by the authors, and in other articles [18], it was observed that the severity of the multipath fading can also change abruptly, together with a modification in the mean value of the received power.

As the channels are uncorrelated in frequency, to simulate protocols that use multiple channels, all the channels need to be simulated simultaneously with their own parameters. In this scenario, switching the channel may improve the QoS of the network when the channel in use presents low quality. More specifically, for each link between two nodes, it is necessary to store 32 values of $P_{R}(d, t)_{\mathrm{dBm}}$ and $K$, which are the parameters of shadowing and fading for the two directions of the link in each of the 16 available channels. Every time a new packet is transmitted in the network, the value of received power is generated considering the parameters stored for the link and channel used in the transmission. This allows capturing the characteristics of uncorrelated channels and the asymmetry of the links. The parameters of the wireless channel are defined as indicated in Table I in the simulation script of Castalia.

The first four parameters are used to calculate the path loss and shadowing, and the parameters $\mathrm{K}$ and $K_{\sigma}$ are used to calculate the small-scale fading. The parameter meanTimeChange defines the mean time of change in the channel characteristics, in minutes. The parameter seed

${ }^{1} \mathrm{http}: / /$ itpp.sourceforge.net/4.3.1/ - Access in 21/12/2016. 
TABLE I

THE PARAMETERS OF THE WIRELESS CHANNEL IN THE SIMULATION SCRIPT OF CASTALIA.

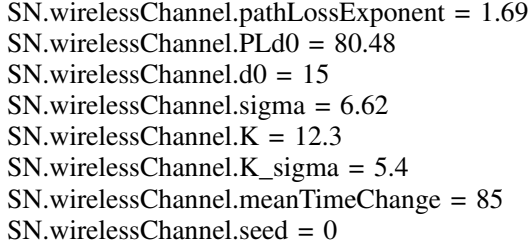

defines the seed used to generate the random values of received power during the simulation. If the value of seed is set to 0 a different seed is defined for each simulation, otherwise the same sequence of values is generated for different simulations. The use of a fixed seed is useful to compare different approaches with the same channel condition.

\section{RESUlts}

The model was integrated into Castalia simulator. At physical layer, it uses real parameters of IEEE 802.15.4 radios, and a model based on log-normal shadowing. The simulator also allows temporal variation in the received power using predefined samples that simulate the behavior of a channel subject to multipath fading. However, by default the model implemented by the simulator considers that the channel is stationary, i.e., its characteristics remain unchanged during all the time. To perform the integration with Castalia, modifications were made in the WirelessChannel class, to capture the variations in the channel characteristics over the long run. After the integration, a simulation was performed, considering one transmitter and one receiver. The simulation parameters are detailed in Table II. The adopted mean time of change seemed to be consistent with the experimental results of [3] [18] [19], in which the channel remains stationary for some hours.

For log-normal shadowing and Rice models, values obtained from experiments in an industrial environment described in [1] were used, considering a scenario without LOS between transmitter and receiver. A transmission power of $0 \mathrm{dBm}$ was used, since it is the transmission power of the radios used in the experiments described in Sections II-A and II-B. The packet transmission rate of 0.2 packets/s is enough for some applications, such as temperature monitoring.

Figure 7 shows the reception power at the receiver (obtained from received data packets) and at the transmitter (obtained from received acknowledgment packets - ACK) during the first four hours of simulation. It is possible to notice the abrupt changes that occur in the channel quality over time, and the asymmetry between the two directions of the link. This type of behavior is realistic in dynamic environments, as is the case of industrial environments. The results obtained by the model are consistent with results from experiments performed in an industrial environment by the authors, and other experimental results described in papers that analyzed the channel behavior for a long period [3] [18] [19], as demonstrated in Section II-A.

Channel 11 was used to obtain the plot shown in Figure 7. Seven additional replications of the simulation were
TABLE II

PARAMETERS USED IN THE SIMULATION.

\begin{tabular}{|l|l|}
\hline Distance between the nodes & $20 \mathrm{~m}$ \\
\hline Physical and MAC layer & $\begin{array}{l}\text { IEEE } 802.15 .4 \text { - CSMA/CA } \\
\text { (without retransmission) }\end{array}$ \\
\hline Simulation Time & $18000 \mathrm{~s}(5 \mathrm{~h}$ ) \\
\hline Transmission power & $0 \mathrm{dBm}$ \\
\hline Packet transmission rate & $0.2 \mathrm{packets} / \mathrm{s}$ \\
\hline Mean time of change & $85 \mathrm{~min}$ \\
\hline Path loss exponent $(n)$ & 1.69 \\
\hline Reference distance for path loss $\left(d_{0}\right)$ & $15 \mathrm{~m}$ \\
\hline Path loss in the reference distance $\left(L\left(d_{0}\right)\right)$ & $80.48 \mathrm{~dB}$ \\
\hline Standard deviation of shadowing $\left(X_{\sigma}\right)$ & $8.13 \mathrm{~dB}$ \\
\hline Ricean factor $(K)$ & $12.3 \mathrm{~dB}$ \\
\hline Ricean factor standard deviation $\left(K_{\sigma}\right)$ & $5.4 \mathrm{~dB}$ \\
\hline
\end{tabular}

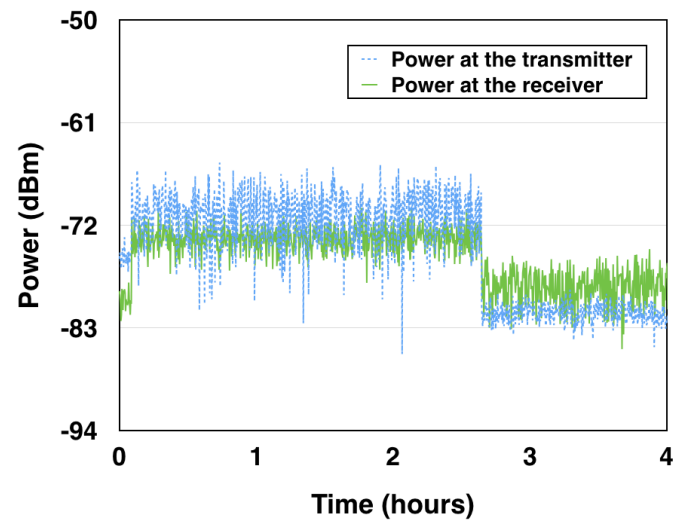

Fig. 7. Asymmetry in the link and temporal variations in the received power.

performed, using different channels, and with the same seed. Figure 8 shows the received power at the receiver for the eight different channels considered. The first 100 samples obtained from each replication were used to plot the chart. The values were filtered using an average filter to better analyze the shadowing in each channel, and to allow a better comparison to the experimental result described in Section II. The results shown in Figures 7 and 8 are similar to the experimental results shown in Figures 1 and 3.

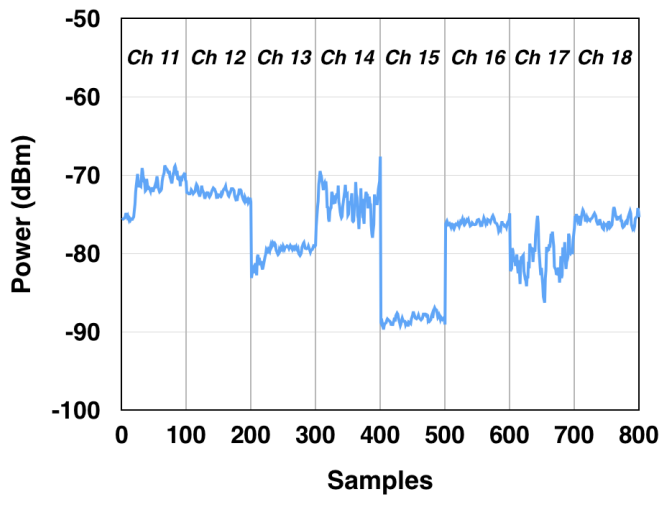

Fig. 8. Received power for different channels. 


\section{A. Evaluation of TMCP Protocol}

TMCP protocol was evaluated to verify how it operates in the presence of dynamic variations in the channels characteristics in the long run. Three different scenarios (A, B and C) were created in the simulator, as shown in Table III. An area of $200 \times 200$ square meters was used, and the end-nodes were randomly placed inside this area. When an end-node cannot reach the sink-node directly, it can use intermediate routers. For example, in Scenario C, routers were assigned by TMCP to the Nodes 12, 22, and 24.

For scenarios that used two channels, two sink-nodes were located in the center of the area, representing a single sink-node with two network interfaces, each one using a different channel (Castalia does not implement nodes with multiple network interfaces). For scenarios with four and eight channels, four and eight sink-nodes were located in the center of the area, respectively. The communication range of the nodes was defined as $70 \mathrm{~m}$ in the execution of TMCP algorithm for the simulations described in this paper. As in [9], the interference range was defined as 1.5 times the communication range $(105 \mathrm{~m})$. This is close to the theoretical communication range of IEEE 802.15.4 radios with $0 \mathrm{dBm}$ of output power.

TABLE III

SCENARIOS FOR SIMULATION.

\begin{tabular}{|c|c|c|c|c|}
\hline Area & Scenario & $\begin{array}{c}\text { Number of } \\
\text { Channels }\end{array}$ & $\begin{array}{c}\text { Total Number of } \\
\text { Nodes }\end{array}$ & $\begin{array}{c}\text { Number of } \\
\text { Sink Nodes }\end{array}$ \\
\hline \multirow{3}{*}{$200 \times 200 \mathrm{~m}$} & $\mathrm{~A}$ & 2 & 21 & 2 \\
\cline { 2 - 5 } & $\mathrm{B}$ & 4 & 23 & 4 \\
\cline { 2 - 5 } & $\mathrm{C}$ & 8 & 27 & 8 \\
\hline
\end{tabular}

Simulations were performed for the three different scenarios using the proposed non-stationary model, and the default stationary model of Castalia. The parameters defined in Table II were also used in these simulations, except the distance between the nodes. Five replications were performed for each scenario.

Figure 9 shows the mean value of PRR considering all nodes connected with each sink-node, for all scenarios. To calculate the mean values, the average PRR was sampled every 10 minutes of simulation, and considering five replications. The confidence level was $99 \%$.

When evaluating the network performance with the stationary model, it is possible to notice that, on average, the performance of the network increases when more channels are used. The overall PRR of the network when using the stationary model was $30.15 \%, 40.8 \%$, and $47.49 \%$, for the scenarios with two, four, and eight channels, respectively. This is explained by the reduction in the collisions between neighbor nodes when more channels are used.

When using the non-stationary model, the overall PRR of the network was $30.34 \%, 31.63 \%$, and $30.66 \%$, for the scenarios with two, four, and eight channels, respectively. Thus, the overall performance remains almost constant for all scenarios when using the non-stationary model, although for some nodes the performance increased in scenarios with more channels. This is a result of the variations that occur
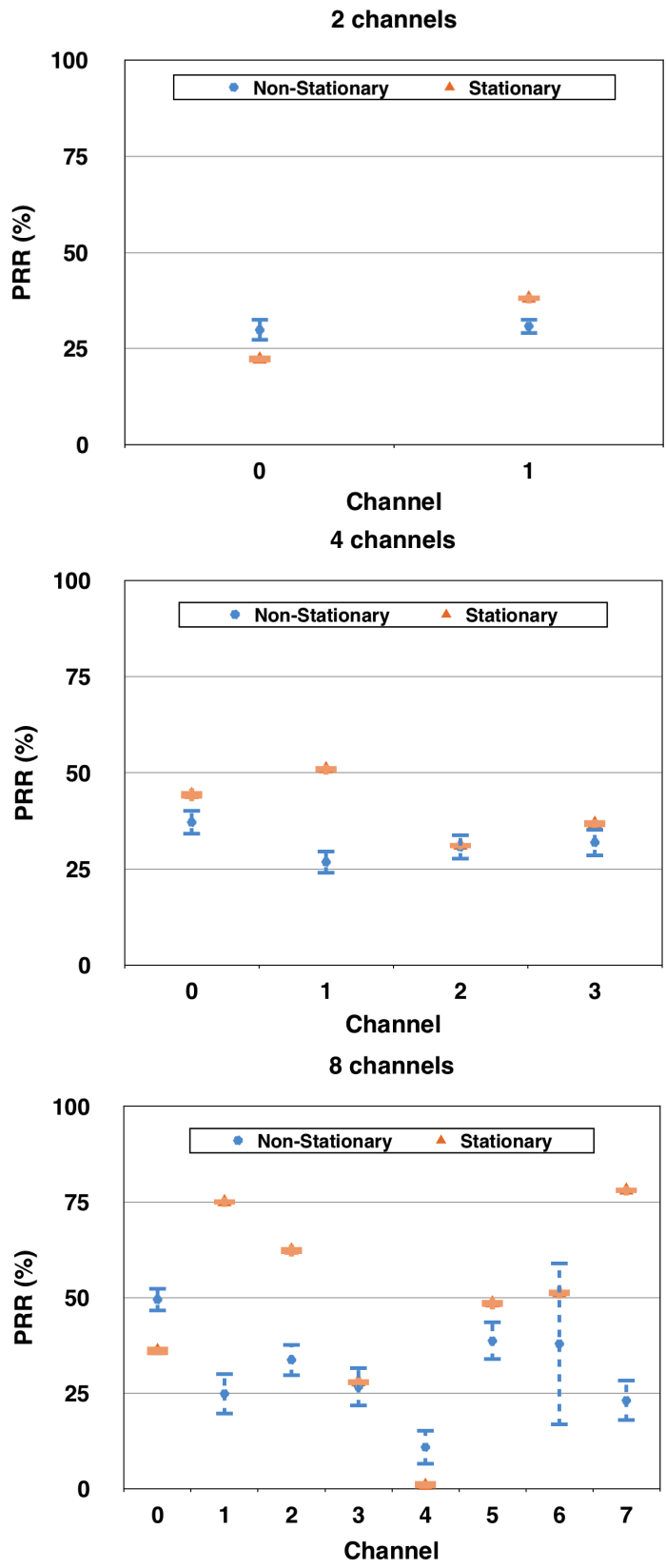

Fig. 9. Averaged values of PRR for each sink-node in all scenarios.

in the channel quality for different links. Even the subtrees have almost the same number of end-nodes, the distances between the end-nodes and the sink-nodes can vary in different sub-trees, and the characteristics of different channels are different for the non-stationary model. Thus, the stationary model may overestimate the network performance.

The most important difference between the two models can be seen in the values of variance. When using the stationary model, the PRR for all sink-nodes presents low values of the variance during five hours of simulation, and even considering five replications, since the nodes are placed in fixed positions. When using the non-stationary model, a higher variance can be observed due to the variation in the channels characteristics over time. The variance is higher when using eight channels. 
This occurs because there are fewer nodes per sub-tree in this scenario. Thus, there is a higher heterogeneity in the characteristics of the sub-trees.

In the scenario that uses two channels, for example, half of the nodes are allocated to a channel and the other half to the other channel. Thus, the variations of a given link can be canceled by an opposite variation in another link inside the sub-tree, which decreases the variation of the average value of PRR considering the whole sub-tree.

In the scenario with eight channels, the PRR for the sub-tree that used channel 4 was nearly zero when using the stationary model. This occurred because the shadowing calculated in the beginning of the simulation by the stationary model remained the same during all time. When considering the non-stationary model, due to the variations in channel quality over time (which can increase or decrease channel quality), the average PRR was higher, and with a higher variance.

Although the averaged values of PRR provide a general assessment of the network performance, it is important to verify the performance of the individual links. For example, for some applications, a minimum PRR needs to be ensured during all time for a specific link. Using the communication between the node [14] and the node [5] (sink-node) as example, the reception power and PRR are illustrated in Figure 10, for the stationary model in (a) and (b), and the non-stationary model in (c) and (d).

To plot the charts in Figure 10, the reception power of the lost packets was fixed at $-100 \mathrm{dBm}$. In Figure $10(\mathrm{c})$, it is possible to see the abrupt changes that occurred in the channel characteristics over time, and the corresponding differences in the PRR (Figure 10(d)), when using the non-stationary model. For the same scenario, and using the stationary channel model of Castalia, there is no change in the channel characteristics during the five hours of simulation, as can be seen in Figure 10(a), and the mean value of PRR also remained constant during all the simulation, as can be seen in Figure 10(b).

The distribution of errors over time was very different for the two models. When using the stationary model, the PRR remained concentrated around 70\% during all simulation. For the non-stationary model, the PRR presented a higher variation, including periods with a very high PRR, and periods with a very low PRR. Figure 11 shows the CDF of the PRR values during all simulation for the two channel models. When using the stationary model, the PRR was greater than $40 \%$ in almost all the simulation. On the other hand, when using the non-stationary model, the PRR remained below $40 \%$ during $20 \%$ of the time.

When the PRR is very low many packets are lost, even with the use of packet retransmission. Besides, the delay and power consumption increase when many retransmission attempts are made. Thus, when considering a non-stationary environment, a static protocol, such as TMCP, is not capable to deal with the variations in the quality of the channels over time, which encourages the development of dynamic channel allocation mechanisms. In the next sub-section, a dynamic protocol, based on TSCH mode of IEEE 802.15.4e standard, is evaluated. Other approaches will be also investigated in future
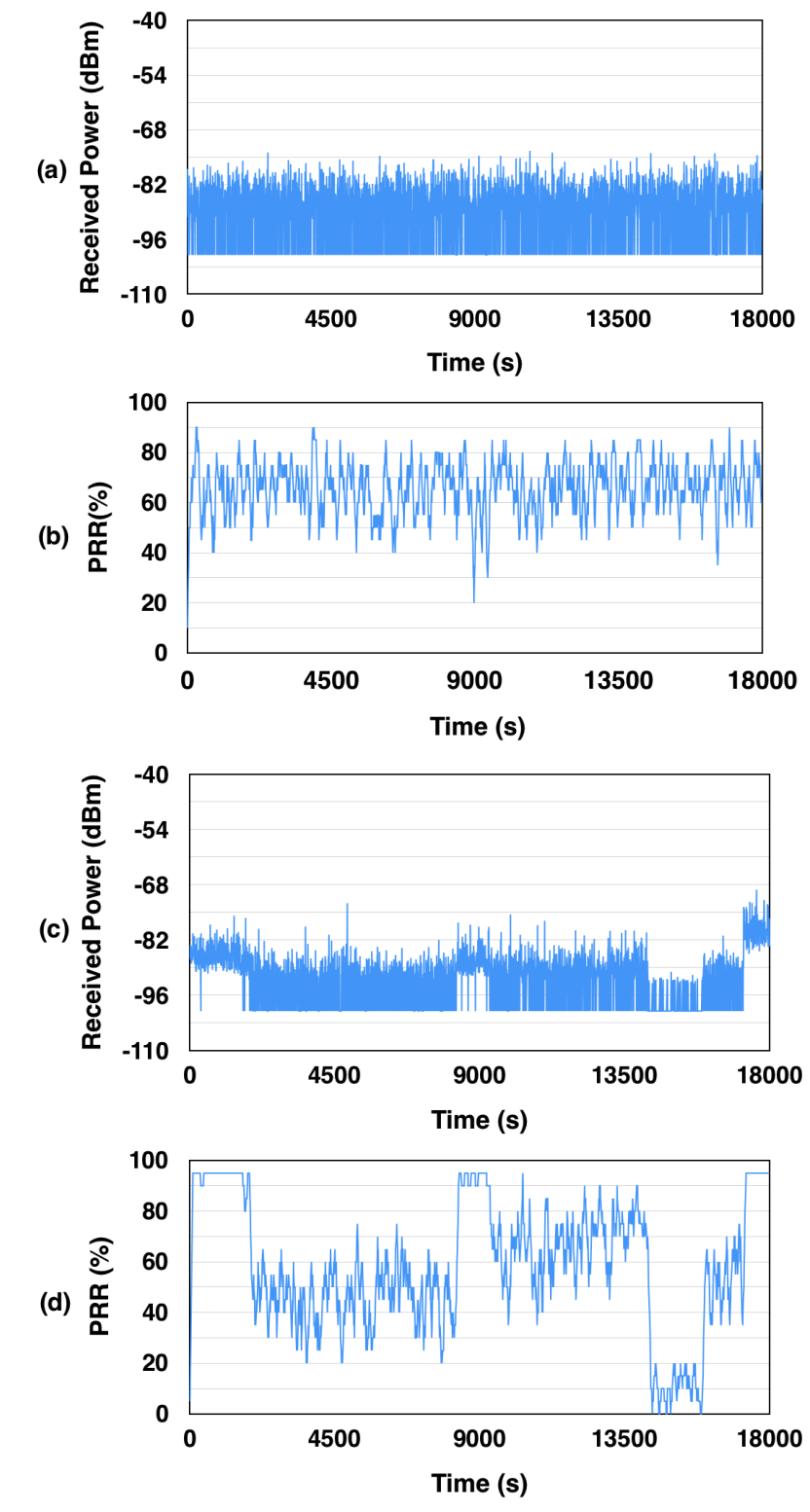

Fig. 10. Received Power (a), and PRR (b) during 5 hours for the link between node [14] and node [5] with the stationary model. Received Power (c), and PRR (d) during the same time and same nodes with the non-stationary model.

works of this research.

\section{B. Comparison between CSMA/CA and a TSCH-based protocol}

This section describes a simulation study to compare the single-channel CSMA/CA protocol and a protocol based on TSCH mode of the IEEE 802.15.4e standard, using the simulation model proposed in this paper. The later uses TDMA and channel hopping, and is similar to MAC protocols defined by WirelessHART and ISA100.11a standards.

In CSMA/CA protocol, when a node has a packet to transmit, it waits for an initial random period (backoff period) before it attempts the transmission, which only occurs if the communication medium is idle. Otherwise, the device waits for another backoff period before trying to access the channel 


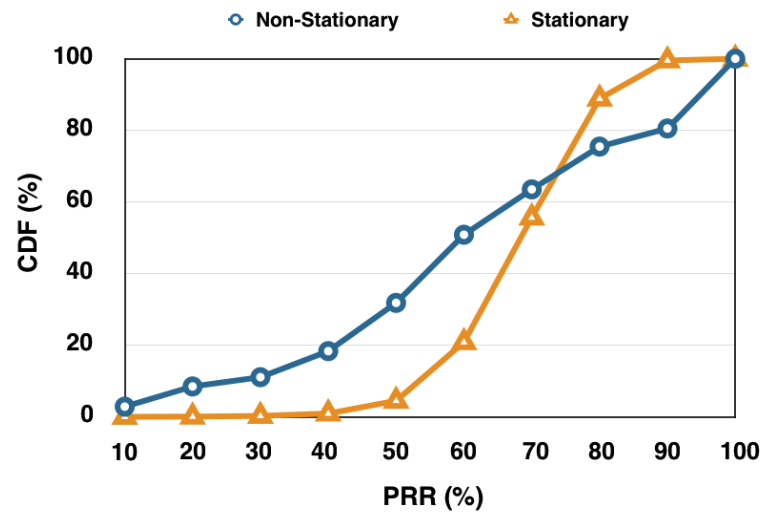

Fig. 11. CDF of the PRR values during all simulation for the link between the node[14] and node[5].

again [28]. If the channel access fails for a given number of attempts (three for the implementation used in this paper), the packet is discarded. In the simulations performed for this paper, the implementation of CSMA/CA algorithm for Castalia simulator described in [29] was used, with a little modification, to allow packet retransmission at MAC layer.

In TSCH, the communication occurs based on a slot-frame structure. A slot-frame is composed by a set of time-slots, and each one is dedicated to one or more nodes. It is possible to have simultaneous transmissions in the same time-slot, in which each transmitter transmits to a different receiver, and using a different channel. When a pair of time-slot and channel is dedicated to only one node, the communication medium can be accessed directly inside the time-slot, without contention, otherwise the nodes use a modified CSMA/CA algorithm to access the channel, to reduce collisions [6]. The slot-frame automatically repeats, and all nodes have a shared notion of time. Typically, the slot allocation is configured by a higher layer when the device joins the network. The nodes hop over the entire channel space to minimize the negative effects of multipath fading and interference [30].

Figure 12 shows an example of a slot-frame with eight time-slots (S0 to S7). Each time-slot has $10 \mathrm{~ms}$ of duration, and accommodates one packet transmission and the corresponding ACK packet. If a given transmitter does not receive the ACK packet inside the time-slot, it can retransmit that packet in the next time-slot allocated to it.

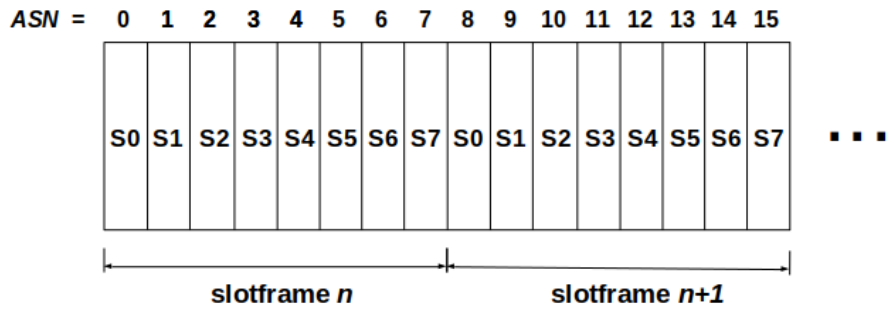

Fig. 12. Example of slot-frame with eight time-slots for a TSCH network.

In the implementation built for the experiments, the channel to be used in a given time-slot is determined using
TABLE IV

POSITION OF THE NODES IN THE SIMULATIONS.

\begin{tabular}{|c|c|c|}
\hline $\begin{array}{c}\text { Node } \\
\text { ID }\end{array}$ & $\begin{array}{c}\text { Coordinates } \\
(\mathbf{X}, \mathbf{Y}, \mathbf{Z})\end{array}$ & $\begin{array}{c}\text { Distance to the } \\
\text { coordinator }(\text { node } \mathbf{0})\end{array}$ \\
\hline 0 & $(7.29,-12.59,2)$ & - \\
\hline 1 & $(13.07,16.51,2)$ & 29.67 meters \\
\hline 2 & $(-2,30,-13.78,2)$ & 9.67 meters \\
\hline 3 & $(-22.65,15.21,2)$ & 40.85 meters \\
\hline 4 & $(21.43,-14.28,2)$ & 14.23 meters \\
\hline 5 & $(-19.45,-9.43,2)$ & 26.93 meters \\
\hline 6 & $(1.78,14.85,2)$ & 27.99 meters \\
\hline 7 & $(-20.70,-2.61,2)$ & 29.72 meters \\
\hline 8 & $(-13.59,24.31,2)$ & 42.40 meters \\
\hline 9 & $(23.79,-9.76,2)$ & 16.74 meters \\
\hline 10 & $(2.28,-10.32,2)$ & 5.50 meters \\
\hline 11 & $(7.70,-8.66,2)$ & 3.95 meters \\
\hline 12 & $(-11,83,-1.79,2)$ & 21.96 meters \\
\hline 13 & $(-18.00,-0.60,2)$ & 27.99 meters \\
\hline 14 & $(-20.83,5.62,2)$ & 33.50 meters \\
\hline 15 & $(-19.93,-14.05,2)$ & 27.26 meters \\
\hline 16 & $(-11.88,-21.59,2)$ & 21.18 meters \\
\hline
\end{tabular}

ch $=\left(A S N+\left\lfloor A S N / S F_{\text {size }}\right\rfloor+\right.$ channelOffset $) \bmod 16$,

in which $A S N$ is the absolute slot number, defined as the total number of time-slots elapsed since the start of the network, and $S F_{\text {size }}$ is the number of time-slots in a slot-frame. A different channelOff set can be defined for each node in the network. Also, the channel $f f$ set is equal to the node ID. Using this formula, the nodes use all the 16 available channels. However, IEEE 802.15.4e standard also defines the use of blacklisting, in which the list of channels considered in the channel hopping mechanism can be reduced. For this simulation study, blacklisting was not considered.

To compare both protocols, the performance of networks in star topology with eight, and 16 end-nodes were evaluated. The end-nodes transmit the packets directly to a sink-node (Node 0). For each configuration, five replications were made, and the performance for each end-node was analyzed individually. The same parameters showed in Table II, regarding the wireless channel, were used. However, for this simulation study, a packet transmission rate of 1 packet/s was used.

To perform a fair comparison, for each replication the same seed was used to evaluate each protocol, and different seeds were used for different replications. Thus, both protocols were evaluated considering the nodes placed at the same position and with the same channel characteristics during the replications. Table IV shows the positions of the nodes, which were determined randomly.

When using CSMA/CA, the end-nodes access the communication medium in a random and distributed way, based on their local notion of the channel availability. Even the end-nodes sense the channel before transmit, packet collisions can occur due to the hidden terminal problem, in which some end-nodes are out of range of other end-nodes. When using $\mathrm{TSCH}$, there are no collisions, since each time-slot is dedicated for only one end-node. In the implementation built for this paper, a slot-frame structure equal to the one illustrated in Figure 12 was used, in which the $S F_{\text {size }}$ is equal to the 


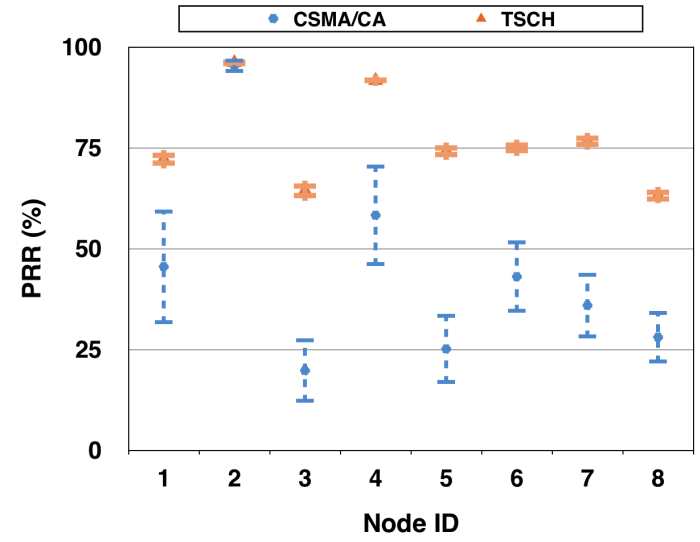

Fig. 13. Averaged values of PRR at MAC layer per end-node for the network with eight end-nodes.

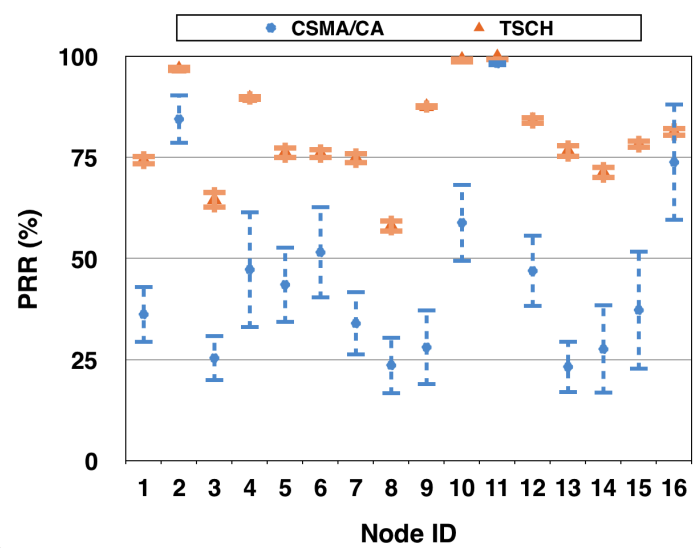

Fig. 14. Averaged values of PRR at MAC layer per end-node for the network with 16 end-nodes.

number of end-nodes in the network. Inside a slot-frame, each end-node has one time-slot dedicated to it. For the scenario with 16 end-nodes, the use of packet retransmission was enabled, with one retransmission attempt per packet in TSCH, and two retransmission attempts in CSMA/CA.

Figures 13, and 14 show the PRR at MAC layer for the networks with eight, and 16 nodes, respectively. As expected, there is a positive correlation between the distance from the end-node to the sink-node, and the PRR for that end-node. When the number of end-nodes increases, the PRR for some end-nodes decreases significantly when using CSMA/CA, due to the higher number of collisions. For example, the PRR of the End Node 4 was approximately $58 \%$, and $47 \%$, for the networks with eight and 16 end-nodes, respectively. On the other hand, when using TSCH, as there are no collisions, the PRR for the End Node 4 was approximately $91,7 \%$, and $90 \%$, for the networks with eight and 16 end-nodes, respectively.

Figure 15 shows the PRR at the application layer, for the network with 16 end-nodes, and using packet retransmission. In this scenario, the average PRR at the application layer, considering all end-nodes, was approximately $76 \%$ and $97 \%$ when using CSMA/CA, and TSCH, respectively. When using TSCH, the lowest PRR was equal to 87\%, for the End Node 8, which is the one with the higher distance to the sink-node.

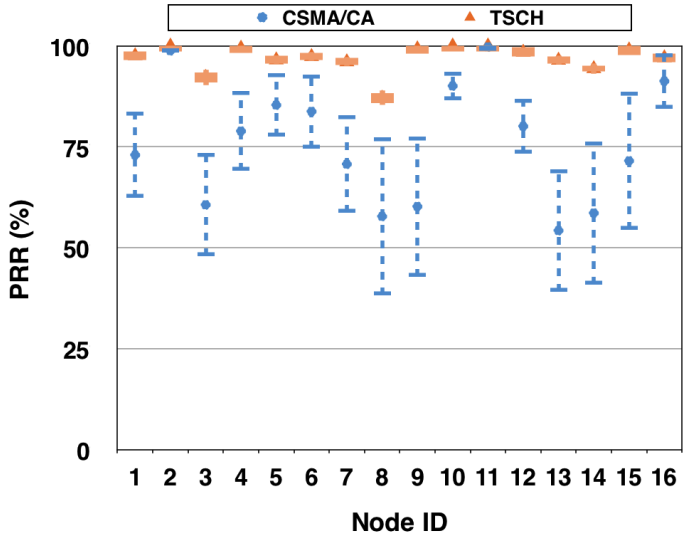

Fig. 15. Averaged values of PRR at the application layer per end-node for the network with 16 end-nodes.

Other aspect to be considered is the high variance of the PRR when using CSMA/CA. When using only one channel, the end-nodes can remain large periods of time with a very low PRR, or even with PRR equal to zero, in cases of deep fading. On the other hand, as in TSCH the end-nodes use all the available channels, the links are less influenced by problems that affect only a subset of channels.

\section{CONCLUSIONS}

This paper presented a reliable simulation model for multi-channel WSN in industrial environments. The proposed model captures the effects of fading, shadowing, and the non-stationary characteristics of the channels in the long run. Besides, the model considers the differences in the characteristics of distinct channels, and the asymmetry of the links. Some results obtained from experimental studies in industrial environments were also presented, to analyze the characteristics of the wireless channel in those environments.

Using the proposed model, the multi-channel protocol TMCP was evaluated, and a comparison study was made between the proposed model and the default model implemented in Castalia simulator. To evaluate the network performance, the PRR and the reception power at the sink-node were used as metrics. It was possible to analyze how the TMCP protocol works in industrial environments. Experiments were conducted to validate the proposed model using three different simulation scenarios, with nodes placed randomly in different positions. The distribution of transmission errors was very different for the two models (stationary and non-stationary), which produced a higher variance for the PRR, when using the non-stationary model. The proposed model captures the actual behavior of the wireless channel in industrial environments.

A static protocol, such as TMCP, is not capable to deal with the variations in the quality of the channels over time. One way to deal with this problem is the use of dynamic channel allocation mechanisms, in which the nodes pick good channels dynamically over time, and not only at the start of the network operation, as is the case for the TMCP protocol.

A simulation study to compare the single-channel protocol CSMA/CA and a TSCH-based protocol, using the proposed 
simulation model, was also described. The results showed that the single-channel CSMA/CA, as proposed in the IEEE 802.15.4 standard, is not adequate to implement the IWSN, since this protocol is not capable to deal with the variations that occur in channel quality over time, neither with the spatial variations in the quality of the channels. Besides, in networks with a high number of nodes, the number of collisions increases, and the performance of the network becomes even worse. Dynamic protocols based on TDMA and frequency hopping, such as MAC protocols proposed by IEEE $802.15 .4 \mathrm{e}$, WirelessHART, and ISA100.11a standards, are more adequate for industrial applications, since the number of collisions is reduced, and the use of channel hopping makes the links more robust to problems that affect only a subset of channels.

Rice distribution was used in this research, but the choice of the best distribution to model fading depends on the type of industrial environment. Other distributions, such as Nakagami- $m$, will be integrated in the simulator in the future. More detailed studies to investigate the performance of dynamic or semi-dynamic multi-channel protocols for IWSN will be performed.

The current implementation of the model for the Castalia simulator, as well as the implementation of TMCP, CSMA/CA, and TSCH for Castalia, can be downloaded from https:/github.com/ruandg/Castalia-IWSN.

\section{ACKNOWLEDGMENT}

The authors would like to thank the support of the Institute for Advanced Studies in Communications (Iecom), the Brazilian Council for Research and Development (CNPq), and the Coordination for the Improvement of Higher Education Personnel (CAPES).

\section{REFERENCES}

[1] E. Tanghe, W. Joseph, L. Verloock, L. Martens, H. Capoen, K. V. Herwegen, and W. Vantomme, "The industrial indoor channel: large-scale and temporal fading at 900, 2400, and $5200 \mathrm{mhz}$," IEEE Transactions on Wireless Communications, vol. 7, no. 7, pp. 2740-2751, July 2008, doi: 10.1109/TWC.2008.070143.

[2] R. D. Gomes, I. E. Fonseca, and M. S. Alencar, "Protocolos multicanais para redes de sensores sem fio industriais," Revista de Tecnologia da Informação e Comunicação, vol. 5, no. 2, pp. 25-32, 2015, doi: 10. 1109/TWC.2008.070143.

[3] P. Agrawal, A. Ahlen, T. Olofsson, and M. Gidlund, "Characterization of long term channel variations in industrial wireless sensor networks," in IEEE International Conference on Communications (ICC), June 2014, pp. 1-6, doi: 10.1109/ICC.2014.6883285.

[4] P. Huang, L. Xiao, S. Soltani, M. Mutka, and N. Xi, "The evolution of mac protocols in wireless sensor networks: A survey," IEEE Communications Surveys \& Tutorials, vol. 15, no. 1, pp. 101-120, First Edition 2013, doi: 10.1109/SURV.2012.040412.00105.

[5] I. Silva, L. A. Guedes, P. Portugal, and F. Vasques, "Reliability and availability evaluation of wireless sensor networks for industrial applications," Sensors, vol. 12, no. 1, pp. 806-838, 2012, doi: 10.3390/ s120100806.

[6] D. D. Guglielmo, S. Brienza, and G. Anastasi, "\{IEEE\} 802.15.4e: A survey," Computer Communications, vol. 88, pp. 1 - 24, 2016, doi: 10.1016/j.comcom.2016.05.004.

[7] R. D. Gomes, G. B. Rocha, A. C. Filho, I. E. Fonseca, and M. S. Alencar, "Distributed approach for channel quality estimation using dedicated nodes in industrial wsn," in 2014 IEEE 25th Annual International Symposium on Personal, Indoor, and Mobile Radio Communication (PIMRC), September 2014, pp. 1943-1948, doi: 10.1109/PIMRC.2014. 7136489.
[8] M. Sha, G. Hackmann, and C. Lu, "Arch: Practical channel hopping for reliable home-area sensor networks," in 17th IEEE Real-Time and Embedded Technology and Applications Symposium, 2011, pp. 305-315, doi: 10.1109/RTAS.2011.36.

[9] W. Du, D. Navarro, and F. Mieyeville, "Performance evaluation of ieee 802.15.4 sensor networks in industrial applications," International Journal of Communication Systems, vol. 28, no. 10, pp. 1657-1674, July 2015, doi: 10.1002/dac.2756.

[10] F. Barac, K. Yu, M. Gidlund, J. Akerberg, and M. Bjorkman, "Towards reliable and lightweight communication in industrial wireless sensor networks," in IEEE 10th International Conference on Industrial Informatics, July 2012, pp. 1218-1224, doi: 10.1109/INDIN.2012. 6300846.

[11] G. Alderisi, G. Patti, O. Mirabella, and L. L. Bello, "Simulative assessments of the ieee $802.15 .4 \mathrm{e}$ dsme and tsch in realistic process automation scenarios," in 2015 IEEE 13th International Conference on Industrial Informatics (INDIN), July 2015, pp. 948-955, doi: 10.1109/ INDIN.2015.7281863.

[12] S. Rekik, N. Baccour, M. Jmaiel, and K. Drira, "Low-power link quality estimation in smart grid environments," in 2015 International Wireless Communications and Mobile Computing Conference (IWCMC), Aug 2015, pp. 1211-1216, doi: 10.1109/IWCMC.2015.7289255.

[13] R. D. Gomes, D. V. Queiroz, I. E. Fonseca, and M. S. Alencar, "Modelo para simulação realista de redes de sensores sem fio industriais," in XXXIII Simpósio Brasileiro de Telecomunicações, 2015, pp. 1-5.

[14] R. D. Gomes, M. S. Alencar, D. V. Queiroz, and I. E. Fonseca, "Evaluation of link quality estimators for industrial wireless sensor networks," in XXXIV Simpósio Brasileiro de Telecomunicações e Processamento de Sinais, 2016, pp. 1-5.

[15] Castalia, "Castalia simulator," access in: 05/12/2016. [Online]. Available: http://castalia.forge.nicta.com.au

[16] T. Rappaport, Wireless Communications: Principles and Practice, 2nd ed. Upper Saddle River, NJ, USA: Prentice Hall PTR, 2001.

[17] T. Olofsson, A. Ahlen, and M. Gidlund, "Modeling of the fading statistics of wireless sensor network channels in industrial environments," IEEE Transactions on Signal Processing, vol. 64, no. 12, pp. 3021-3034, June 2016, doi: 10.1109/TSP.2016.2539142.

[18] P. Agrawal, A. Ahlen, T. Olofsson, and M. Gidlund, "Long term channel characterization for energy efficient transmission in industrial environments," IEEE Transactions on Communications, vol. 62, no. 8, pp. 3004-3014, August 2014, doi: 10.1109/TCOMM.2014.2332876.

[19] N. C. Wang and K. Yao, "Characterizing fading channel under abrupt temporal variations," in 2013 IEEE International Conference on Acoustics, Speech and Signal Processing (ICASSP), May 2013, pp. 5056-5060, doi: 10.1109/ICASSP.2013.6638624.

[20] R. D. Gomes, M. S. Alencar, I. E. Fonseca, and A. C. Lima Filho, "Desafios de redes de sensores sem fio industriais," Revista de Tecnologia da Informação e Comunicação, vol. 4, pp. 1-12, 2014, doi: 10.12721/2237-5112.v04n01a03.

[21] D. M. Amzucu, H. Li, and E. Fledderus, "Indoor radio propagation and interference in $2.4 \mathrm{ghz}$ wireless sensor networks: Measurements and analysis," Wireless Personal Communications, vol. 76, pp. 245-269, 2014, doi: 10.1007/s11277-014-1694-2.

[22] L. O. Varga, M. Heusse, R. Guizzetti, and A. Duda, "Why is Frequency Channel Diversity so Beneficial in Wireless Sensor Networks?" in IFIP Wireless Days. Toulouse, France: IFIP, March 2016, access in: 05/12/2016. [Online]. Available: https: //hal.archives-ouvertes.fr/hal-01287518

[23] T. Watteyne, S. Lanzisera, A. Mehta, and K. S. J. Pister, "Mitigating multipath fading through channel hopping in wireless sensor networks," in IEEE International Conference on Communications (ICC), May 2010, pp. 1-5, doi: 10.1109/ICC.2010.5502548.

[24] O. D. Incel, "A survey on multi-channel communication in wireless sensor networks," Computer Networks, vol. 55, no. 13, pp. 3081 - 3099, 2011, doi: https://doi.org/10.1016/j.comnet.2011.05.020".

[25] Y. Wu, J. Stankovic, T. He, and S. Lin, "Realistic and efficient multi-channel communications in wireless sensor networks," in IEEE INFOCOM 2008 - The 27th Conference on Computer Communications, April 2008, doi: 10.1109/INFOCOM.2008.175.

[26] S. Petersen and S. Carlsen, "Performance evaluation of wirelesshart for factory automation," in 2009 IEEE Conference on Emerging Technologies Factory Automation, Sept 2009, pp. 1-9, doi: 10.1109/ ETFA.2009.5346996.

[27] A. Goldsmith, Wireless Communications. New York, NY, USA: Cambridge University Press, 2005.

[28] IEEE Standard for Information technology - Local and metropolitan area networks - Specific requirements - Part 15.4: Wireless Medium Access 
Control (MAC) and Physical Layer (PHY) Specifications for Low Rate Wireless Personal Area Networks (WPANs), 2006.

[29] U. Colesanti and S. Santini, "The Collection Tree Protocol for the Castalia wireless sensor networks simulator," ETH Zurich, Tech. Rep., June 2011.

[30] "Ieee standard for local and metropolitan area networks-part 15.4: Low-rate wireless personal area networks (lr-wpans) amendment 1 : Mac sublayer," IEEE Std 802.15.4e-2012 (Amendment to IEEE Std 802.15.4-2011), pp. 1-225, April 2012.

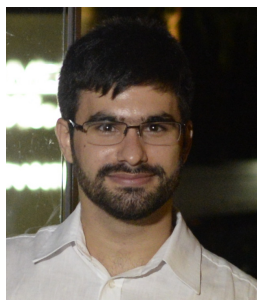

Ruan D. Gomes received his B.S. degree in Computer Science from Federal University of Paraíba, and his M.S. degree in Computer Science from Federal University of Campina Grande (UFCG). He currently works as a Lecturer at the Federal Institute of Education, Science, and Technology of Paraíba, Campus Guarabira. He is also a Ph.D. student in Electrical Engineering at UFCG, and is currently in a doctoral mobility at the Universidad Politécnica de Madrid. He has experience in $R \& D$ projects, mainly in the fields of embedded systems, wireless sensor networks, and multimedia systems.

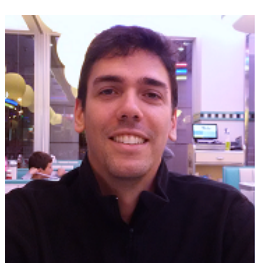

Diego V. Queiroz received his BSc in Computer Science at University Center of João Pessoa (2005) and BSc in Technology of Computer Networks at Federal Institute of Education, Science and Technology of Paraíba (2008). He received his MSc in Informatics from Federal University of Paraíba (UFPB) in 2015, and currently is a Ph.D. student at Universidad Politécnica de Madrid. His research areas include: information security, computer networks, wireless communications and network simulators.

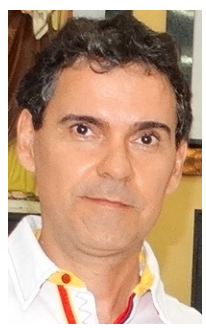

Iguatemi E. Fonseca was born in Brazil, in 1974. He is currently an associate professor at the Informatics Center of the Federal University of Paraíba. He received the electronics engineering degree from the Federal University of Campina Grande (UFCG), Campina Grande, PB, Brazil, in 1999, and the M.Sc. and Ph.D. degrees from the State University of Campinas (Unicamp), Campinas, SP, Brazil, in 2001 and 2005, respectively, specializing in nonlinear fiber optics and its applications, optical network design with physical-layer impairments and Impairment-Aware RWA. His current research interests include areas of the Electrical Engineering and Computer Science as: i) Communications Networks (Optical Networks, Wireless Sensor Networks, Mobile Networks and IP Networks), working on topics as identification/models of traffic in computer networks, techniques for identification and mitigation of denial of service attacks, algorithms and protocols in Industrial Wireless Sensor Networks, WDM and elastic optical networks with QoS requirements, and design and simulation of photonics devices; ii) Design of simulators in virtual reality with applications in industry sectors, as energy sector.

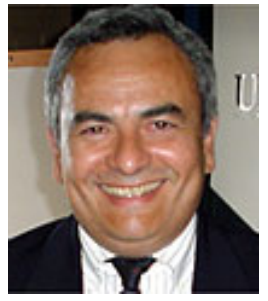

Marcelo S. Alencar was born in Serrita, Brazil in 1957. He received his Bachelor Degree in Electrical Engineering, from Universidade Federal de Pernambuco (UFPE), Brazil, 1980, his Master Degree from Universidade Federal da Paraíba (UFPB), Brazil, 1988 and his Ph.D. from the University of Waterloo, Department of Electrical and Computer Engineering, Canada, 1994. Marcelo $\mathrm{S}$. Alencar has more than 35 years of engineering experience, and 26 years as an IEEE Member, currently as Senior Member. For 18 years he worked for the Department of Electrical Engineering, Federal University of Paraíba, where he was Full Professor and supervised more than 50 graduate and several undergraduate students. Since 2003, he is Chair Professor at the Department of Electrical Engineering, Federal University of Campina Grande, Brazil. He published 20 books and more than 420 papers in journals and conferences. He is columnist of the traditional newspaper Jornal do Commercio, in Recife, Brazil. 